\title{
Debonding criterion for the piezoelectric fibre push-out test
}

\author{
J.-S. WANG† and Q.-H. QIN*\$ \\ $\dagger$ Department of Mechanics, School of Mechanical Engineering, Tianjin University, \\ Tianjin 300072, China \\ †Department of Engineering, Australian National University, \\ Canberra, ACT 0200, Australia
}

(Received 19 June 2005; in final form 27 December 2005)

\begin{abstract}
A theoretical model of piezoelectric fibre push-out is developed within the framework of a modified shear-lag theory to study stress and electrical field transfer. Based on the model, a debonding criterion for the piezoelectric effect is presented utilizing a fracture-mechanics approach to investigate the debonding process of piezoelectric fibre in the push-out test for combined electrical and mechanical loading, and for mechanical loading only. A numerical example is considered to verify the proposed debonding criterion. The results show that stress and electrical fields can be controlled by the interface. The study also shows that interfacial properties and piezoelectric coefficients have a significant influence on the debonding process of piezoelectric fibre composites, and the energy-release rate can be enhanced or reduced depending on the direction of electrical loading, which shows that the energy-release rate can be used as the debonding criterion.
\end{abstract}

\section{Introduction}

Piezoelectric fibre composites (PFCs), which comprise uniaxially aligned piezoelectric fibres embedded in a polymer matrix, have been widely used in recent years as transducers in applications such as sensors and actuators, sonar projectors, underwater use, medical ultrasonic imaging and health-monitoring systems [1]. PFCs combine the advantages of the electromechanical performance of piezoelectric fibres with the excellent toughness and flexibility of a polymer matrix, thus, exhibiting significant advantages over monolithic piezoelectric materials. These advantages include anisotropic actuation and sensing, the ability to overcome low fracture strength and conform to curved surfaces, among others [2,3]. With the rapid development in piezoelectric fibre and polymer manufacturing techniques, as well as their wider application in industry, there has been increasing interest in the study of piezoelectric composites and their electrical and mechanical performance under service conditions.

Despite the wide application of PFCs in practical engineering, many critical issues relating to PFCs are yet to be resolved. Such issues include the understanding

*Corresponding author. Email: qinghua.qin@anu.edu.au 
and characterization of coupled electrical and mechanical behavior, interface properties between piezoelectric fibre and matrix, load and electrical field transfer mechanisms between fibre and matrix, and interfacial debonding mechanisms. It should be noted that improvement in the fracture toughness of PFCs depends heavily on the polymer matrix, which is very different from conventional (nonpiezoelectric) fibre-reinforced composites (FRCs), although the interfacial load transfer mechanism in PFCs is similar to that in FRCs. In addition, interfacial properties have a significant effect on the distribution of the electrical field in piezoelectric fibres. As such, the mechanical behaviour of the interface has a critical influence on the electromechanical performance and fracture behavior of PFCs. Thus, interfacial properties constitute a key issue in the design and application of PFCs. Despite the importance of interfacial behavior in PFCs, study of the interfacial debonding process has progressed quite slowly owing to the complexity of the problem and lack of experimental data. Recently, Liu et al. [4] presented a theoretical model of piezoelectric fibre pull-out to study the interaction between fibre deformation, pull-out stress and the corresponding electrical field using a shear stress criterion. Qin et al. [5] used the method described by Liu et al. [4] to investigate the effect of the piezoelectric coefficients and fibre length on the stress fields and electrical field for a fully bonded and a fully debonded interface, and compared their results to those for non-piezoelectric fibre composites.

In contrast, in non-piezoelectric FRCs, the interfacial debonding problem has been extensively investigated through many analytical models, such as shearlag-based models. Using a shear strength criterion, Hsueh [6, 7] presented an energy-based approach for determining the debonding behavior of nonpiezoelectric fibre composite in a pull-out/push-out test. Gao et al. [8] established a debonding criterion using the concept of fracture mechanics [9]. During the process of push-out or pull-out, fibre debonding may take place in a mixed mode rather than in a pure shear mode and, in this case, the shear stress criterion may not be applicable as it does not incorporate the effect of tensile radial stress on interfacial failure [10]. To address this problem, an interfacial debonding criterion was developed, based on the concept of fracture mechanics, to analyse the debonding process in a frictional pull-out test [9]. It is noted that shear-lag theory is traditional and well-established, but the existing theory is suitable for elastic (non-piezoelectric) fibre composites only and cannot be directly applied to piezoelectric fibre composites for characterizing electromechanical behavior of the interface in PFCs, since eletromechanical coupling behavior is not incorporated in the existing models. It is, therefore, desirable to develop a theoretical model and an interfacial debonding criterion, specifically considering the piezoelectric effect, to investigate the piezoelectric fibredebonding process in push-out and pull-out tests. This is the rationale behind the study.

The purpose of this study is to extend previous analyses to incorporate the effect of electromechanical coupling in the piezoelectric fibre push-out test. A modified shear-lag theory, which takes into consideration the piezoelectric effect and the inverse piezoelectric effect, is developed to construct a debonding criterion of piezoelectric composites. 
Based on this modified shear-lag theory, an interface debonding energy functionally incorporating the electroelastic effect is developed to characterize debonding behavior, and can be used as a debonding criterion in push-out problems. It should be pointed out that the Liu et al. model [4] was based on the shear stress criterion, which essentially follows the procedure in [11]. In contrast, the proposed model is based on an electroelastic energy criterion. In addition, in our model, the expressions for $\sigma_{m}^{r}$ and $\sigma_{m}^{\theta}$ do not include the term $d \tau_{i} / d z$, which was used in the Liu et al. model [4]. In fact, the present model extends Zhou et al.'s model [9] to include the piezoelectric effect, which is distinctly different from [11]. Besides, the Liu et al. model cannot handle the case of electrical loading. The energyrelease rate, the stress field and electrical field induced by the deformation and the electrical loading in the debonding and bonding region of the piezoelectric fibre push-out model are obtained within the framework of shear-lag theory. The piezoelectric effects and the effect of electrical loading on stress fields and debonding behaviour are extensively investigated using the proposed model and the results are compared with those for different electrical loading. Numerical examples are considered to study the effect of interfacial property parameters, such as the frictional coefficient $\mu$, the residual compressive stress $q_{0}$, and the applied electrical loading, on the distribution of the electrical field in fibres. Using the proposed energy-release rate, including electromechanical coupling, the variation of the normalized energy release rate against the normalized debonding length $l / L$ are presented, providing some initial insights into the piezoelectric fibre debonding process.

\section{Theoretical model}

The geometric configuration of the micromechanical model used in the single piezoelectric fibre push-out test is shown in figure 1, with an interfacial debonding crack of length $l$. A piezoelectric fibre, polarized in the axial direction with radius $a$ and length $L$, is embedded at the centre of a coaxial cylindrical shell of epoxy matrix with external radius $b$. A uniform compressive stress $\sigma_{a}$ and an electrical loading $V$ are applied at the fibre end $(z=0)$. The piezoelectric fibre is considered transversely isotropic and the epoxy is isotropic. In the following, theoretical models are derived for the case of mechanical and electromechanical loading.

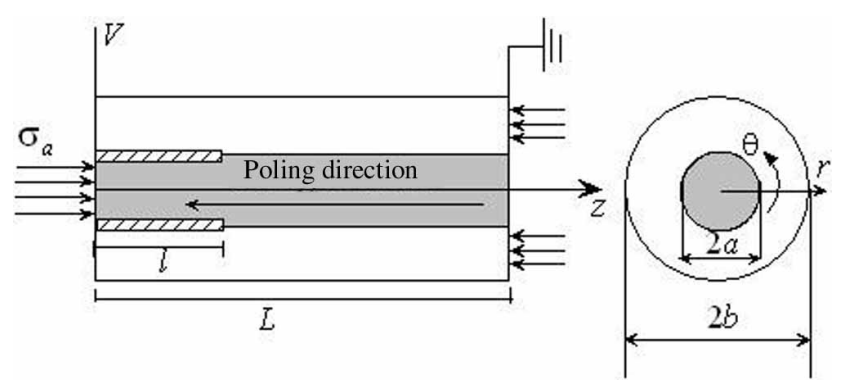

Figure 1. Piezoelectric fibre/matrix cylinder model in the fibre push-out test. 


\subsection{Mechanical loading}

Based on linear piezoelectric theory, the relationship between strains and stresses is expressed as $[12,13]$ :

$$
\begin{gathered}
{\left[\begin{array}{c}
\varepsilon_{f}^{r} \\
\varepsilon_{f}^{\theta} \\
\varepsilon_{f}^{z} \\
2 \varepsilon_{f}^{r z}
\end{array}\right]=\left[\begin{array}{cccc}
s_{11} & s_{12} & s_{13} & 0 \\
s_{12} & s_{11} & s_{13} & 0 \\
s_{13} & s_{13} & s_{33} & 0 \\
0 & 0 & 0 & s_{55}
\end{array}\right]\left[\begin{array}{c}
\sigma_{f}^{r} \\
\sigma_{f}^{\theta} \\
\sigma_{f}^{z} \\
\sigma_{f}^{r z}
\end{array}\right]+\left[\begin{array}{cc}
0 & g_{13} \\
0 & g_{13} \\
0 & g_{33} \\
g_{15} & 0
\end{array}\right]\left[\begin{array}{c}
D^{r} \\
D^{z}
\end{array}\right]} \\
{\left[\begin{array}{c}
E^{r} \\
E^{z}
\end{array}\right]=-\left[\begin{array}{cccc}
0 & 0 & 0 & g_{15} \\
g_{13} & g_{13} & g_{33} & 0
\end{array}\right]\left[\begin{array}{c}
\sigma_{f}^{r} \\
\sigma_{f}^{\theta} \\
\sigma_{f}^{z} \\
\sigma_{f}^{r z}
\end{array}\right]+\left[\begin{array}{cc}
e_{11} & 0 \\
0 & e_{33}
\end{array}\right]^{-1}\left[\begin{array}{l}
D^{r} \\
D^{z}
\end{array}\right]}
\end{gathered}
$$

where $\varepsilon, \sigma, E$ and $D$ are, respectively, strain and stress tensors, electrical field strength and electrical displacement vectors. The superscripts $r, \theta$ and $z$ refer to the radial, circumferential, and axial directions, respectively in a cylindrical coordinate system. In the above formula, $g_{i j}$ and $e_{i j}$ are piezoelectric coefficient $\left(\mathrm{VmN}^{-1}\right)$ and dielectric constants $\left(\mathrm{NV}^{-2}\right), s_{i j}$ are components of elastic compliance.

The electrical field $E$ is defined by

$$
E^{i}=-\Phi_{, i}
$$

where $\Phi$ is the electrical potential and $i$ refers to $r$ and $z$.

The electrical displacements satisfy the following equation:

$$
\frac{\partial D^{r}}{\partial r}+\frac{D^{r}}{r}+\frac{\partial D^{z}}{\partial z}=0
$$

The equilibrium between the axial and interfacial stress can be expressed as:

$$
\begin{aligned}
& \sigma_{a}=\sigma_{f}^{z}+\frac{1}{\gamma} \sigma_{m}^{z} \\
& \frac{d \sigma_{f}^{z}}{d z}=-\frac{2}{a} \tau_{i}(z)
\end{aligned}
$$

where

$$
\gamma=a^{2} /\left(b^{2}-a^{2}\right)
$$

The analytical solution of the boundary-value problem defined by equations (1)-(6) is difficult to obtain for the fibre push-out problem under consideration. Hence, an approximate solution within the shear-lag framework is sought here, with certain assumptions. To simplify the analysis, it is assumed that the whole body is in a plane strain state. The electrical potential in the fibre is taken as the average in the $r$-direction, so it is independent of $r$, and the axial stresses are treated 
similarly. Thus, the axial stresses and electrical potential on any cross-section of the composite can be written in the form:

$$
\sigma_{f}^{z}=\sigma_{f}^{z}(z), \quad \sigma_{m}^{z}=\sigma_{m}^{z}(z), \quad \Phi=\Phi(z)
$$

For a long fibre $(L \gg a)$ polarized in the $z$-direction embedded in a relatively large matrix, this assumption is appropriate and, due to the transversely isotropic property of the piezoelectric fibre, the following assumption is still acceptable:

$$
\sigma_{f}^{r}(z)=\sigma_{f}^{\theta}(z)=q_{i}(z)
$$

in which $q_{i}(z)$ is the interfacial radial stress due to Poisson contraction between the fibre and the matrix. Then, from equations (2), (5) and (8), (9), we can obtain the following formula [4]:

$$
D^{z}=d_{15} \sigma_{f}^{z}
$$

In the debonding region, stress transfer is governed by the Coulomb friction law:

$$
\tau_{i}(z)=-\mu\left[q_{0}-q_{i}(z)\right]
$$

in which $\mu$ is a constant coefficient of friction and $q_{0}$ is the residual compressive stress during the manufacturing process.

Using the mechanical boundary conditions and a procedure similar to that in [9], the stress fields in the debonded region $(0 \leq z \leq l)$ are obtained as:

$$
\begin{gathered}
q_{i}(z)=-\frac{E_{m}\left(s_{13}+g_{31} d_{15}\right) \sigma_{f}^{z}+v_{m} \sigma_{m}^{z}}{E_{m}\left(s_{11}+s_{12}\right)+1+2 \gamma+v_{m}} \\
\sigma_{m}^{z}(z)=\gamma \omega\left[\sigma^{*}+\sigma_{a}\right][1-\exp (-\lambda z)] \\
\sigma_{f}^{z}(z)=\sigma_{a}-\omega\left[\sigma^{*}+\sigma_{a}\right][1-\exp (-\lambda z)] \\
\tau_{i}(z)=\frac{a \lambda \omega}{2}\left(\sigma^{*}+\sigma_{a}\right) \exp (-\lambda z)
\end{gathered}
$$

in which

$$
\begin{gathered}
\kappa=-\frac{E_{m}\left(s_{13}+g_{31} d_{15}\right)-\gamma v_{m}}{E_{m}\left(s_{11}+s_{12}\right)+1+2 \gamma+v_{m}} \\
\omega=\frac{E_{m}\left(s_{13}+g_{31} d_{15}\right)}{E_{m}\left(s_{13}+g_{31} d_{15}\right)-\gamma v_{m}} \\
\lambda=\frac{2 \mu \kappa}{a} \\
\sigma^{*}=-\frac{q_{0}}{\omega \kappa}
\end{gathered}
$$

where $E_{m}, v_{m}$ are Young's modulus and Poisson's ratio of the matrix, respectively. 
The solutions of the stress fields in the bonded region $(l \leq z \leq L)$ are given by:

$$
\begin{gathered}
\sigma_{f}^{z}(z)=\frac{\left(\frac{A_{2}}{A_{1}} \sigma_{a}+\sigma_{l}\right) \sinh \sqrt{A_{1}}(L-z)-\frac{A_{2}}{A_{1}} \sigma_{a} \sinh \sqrt{A_{1}}(l-z)}{\sinh \sqrt{A_{1}}(L-l)}-\frac{A_{2}}{A_{1}} \sigma_{a} \\
\sigma_{m}^{z}(z)=\gamma\left(1+\frac{A_{2}}{A_{1}}\right) \sigma_{a}-\gamma \frac{\left(\frac{A_{2}}{A_{1}} \sigma_{a}+\sigma_{l}\right) \sinh \sqrt{A_{1}}(L-z)-\frac{A_{2}}{A_{1}} \sigma_{a} \sinh \sqrt{A_{1}}(l-z)}{\sinh \sqrt{A_{1}}(L-l)} \\
\tau_{i}(z)=\frac{\lambda a\left[\left(\frac{A_{2}}{A_{1}} \sigma_{a}+\sigma_{l}\right) \cosh \sqrt{A_{1}}(L-z)-\frac{A_{2}}{A_{1}} \sigma_{a} \cosh \sqrt{A_{1}}(l-z)\right]}{2 \sinh \sqrt{A_{1}}(L-l)}
\end{gathered}
$$

where

$$
\begin{gathered}
\sigma_{l}=\sigma_{f}^{z}(l)=\sigma_{a}-\omega\left[\sigma^{*}+\sigma_{a}\right][1-\exp (-\lambda l)] \\
A_{1}=\frac{2\left[\gamma+E_{m}\left(s_{33}+g_{33} d_{15}\right)-2 \kappa\left(\gamma v_{m}-E_{m} s_{13}\right)\right]}{\left(1+v_{m}\right)\left[2 \gamma b^{2} \ln (b / a)-a^{2}\right]} \\
A_{2}=-\frac{2\left[\gamma+2\left(\gamma v_{m}-E_{m} s_{13}\right)(\omega-1) \kappa\right]}{\left(1+v_{m}\right)\left[2 \gamma b^{2} \ln (b / a)-a^{2}\right]}
\end{gathered}
$$

The electrical field $E^{z}$ in both the debonded and bonded regions is given as:

$$
E^{z}=-2 g_{31} \kappa(\omega-1) \sigma_{a}+\left[\frac{d_{15}}{e_{33}}-g_{33}-2 g_{31} \kappa\right] \sigma_{f}^{z}
$$

\subsection{Electromechanical loading}

In this case, the constitutive equations for the piezoelectric fibre polarized along the $z$-axis can be written as [12]:

$$
\begin{aligned}
& {\left[\begin{array}{c}
\varepsilon_{f}^{r} \\
\varepsilon_{f}^{\theta} \\
\varepsilon_{f}^{z} \\
2 \varepsilon_{f}^{r z}
\end{array}\right]=\left[\begin{array}{cccc}
s_{11} & s_{12} & s_{13} & 0 \\
s_{12} & s_{11} & s_{13} & 0 \\
s_{13} & s_{13} & s_{33} & 0 \\
0 & 0 & 0 & s_{55}
\end{array}\right]\left[\begin{array}{c}
\sigma_{f}^{r} \\
\sigma_{f}^{\theta} \\
\sigma_{f}^{z} \\
\tau_{f}^{r z}
\end{array}\right]+\left[\begin{array}{cc}
0 & d_{13} \\
0 & d_{13} \\
0 & d_{33} \\
d_{15} & 0
\end{array}\right]\left[\begin{array}{c}
E^{r} \\
E^{z}
\end{array}\right]} \\
& {\left[\begin{array}{c}
D^{r} \\
D^{z}
\end{array}\right]=\left[\begin{array}{cccc}
0 & 0 & 0 & d_{15} \\
d_{13} & d_{13} & d_{33} & 0
\end{array}\right]\left[\begin{array}{c}
\sigma_{f}^{r} \\
\sigma_{f}^{\theta} \\
\sigma_{f}^{z} \\
\tau_{f}^{r z}
\end{array}\right]+\left[\begin{array}{cc}
e_{11} & 0 \\
0 & e_{33}
\end{array}\right]\left[\begin{array}{l}
E^{r} \\
E^{z}
\end{array}\right]}
\end{aligned}
$$


Considering equations (3), (4), (8), (9), (27) and (28), we can deduce that the electrical potential can be obtained and written in the form:

$$
\Phi(z)=\frac{1}{e_{33}} \int_{0}^{z}\left[2 d_{13} q_{i}(z)+\left(d_{33}-d_{15}\right) \sigma_{f}^{z}(z)\right] d z+C_{1} z+C_{2}
$$

in which

$$
C_{1}=-\frac{V}{L}-\frac{1}{e_{33} L} \int_{0}^{L}\left[2 d_{13} q_{i}(z)+\left(d_{33}-d_{15}\right) \sigma_{f}^{z}(z)\right] d z, \quad C_{2}=V
$$

Electrical boundary conditions at the ends of piezoelectric fibre are given as:

$$
\Phi(0)=V, \quad \Phi(L)=0
$$

The mechanical boundary conditions are the same as those in [9].

The solutions for stress distribution in the constituents are obtained in the bonded and debonded regions, and are exactly the same as those given in equations (13)-(15) and (20)-(22), except that some variables and parameters are replaced by the following expressions:

$$
\begin{aligned}
& q_{i}(z)=-\frac{E_{m}\left[s_{13}-d_{13}\left(d_{33}-d_{15}\right) / e_{33}\right] \sigma_{f}^{z}(z)+v_{m} \sigma_{m}^{z}(z)-E_{m} d_{13} C_{1}}{E_{m}\left(s_{11}+s_{12}-2 d_{13}^{2} / e_{33}\right)+1+2 \gamma+v_{m}} \\
& \kappa=-\frac{E_{m}\left[s_{13}-d_{13}\left(d_{33}-d_{15}\right) / e_{33}\right]-\gamma v_{m}}{E_{m}\left(s_{11}+s_{12}-2 d_{13}^{2} / e_{33}\right)+1+2 \gamma+v_{m}} \\
& \omega=\frac{E_{m}\left[s_{13}-d_{13}\left(d_{33}-d_{15}\right) / e_{33}\right]}{E_{m}\left[s_{13}-d_{13}\left(d_{33}-d_{15}\right) / e_{33}\right]-\gamma v_{m}} \\
& q^{*}=\frac{E_{m} d_{13} C_{1}}{E_{m}\left(s_{11}+s_{12}-2 d_{13}^{2} / e_{33}\right)+1+2 \gamma+v_{m}} \\
& \sigma^{*}=-\frac{q_{0}-q^{*}}{\kappa \omega} \\
& \lambda=\frac{2 \mu \kappa}{a} \\
& A_{1}=\frac{2\left\{\gamma+E_{m}\left[s_{33}-d_{33}\left(d_{33}-d_{15}\right) / e_{33}\right]-2 \kappa\left(\gamma v_{m}-E_{m} s_{13}+E_{m} d_{13} d_{33} / e_{33}\right)\right\}}{\left(1+v_{m}\right)\left[2 \gamma b^{2} \ln (b / a)-a^{2}\right]} \\
& A_{2}=-\frac{2\left\{\begin{array}{l}
{\left[\gamma+2\left(\gamma v_{m}-E_{m} s_{13}+E_{m} d_{13} d_{33} / e_{33}\right)(\omega-1) \kappa\right]} \\
+2\left[\gamma v_{m}-E_{m}\left(s_{13}-d_{13} d_{33} / e_{33}\right)\right] q * / \sigma_{a}+E_{m} d_{33} c_{1} / \sigma_{a}
\end{array}\right\}}{\left(1+v_{m}\right)\left[2 \gamma b^{2} \ln (b / a)-a^{2}\right]}
\end{aligned}
$$


The electrical field $E^{z}$ in both the bonded and debonded regions is given as

$$
E^{z}=-\frac{1}{e_{33}}\left[2 d_{13} q_{i}(z)+\left(d_{33}-d_{15}\right) \sigma_{f}^{z}(z)\right]-C_{1}
$$

\section{The interfacial debonding criterion}

Unlike in non-piezoelectric fibre composites, in PFCs there are electrical fields caused by the piezoelectric effect or inverse-piezoelectric effect. Owing to this phenomenon, the debonding criterion for non-piezoelectric fibre composites is not directly applicable. To incorporate the piezoelectric effect in the debonding criterion, we consider a cracked piezoelectric elastic body of volume $V$ in which traction $P$, frictional stress $t$, and the surface electrical charge $\omega$ are applied. $S_{P}, S_{t}$ and $S_{\omega}$ are the corresponding surfaces, respectively, as shown in figure 2. For the sake of simplicity, the matrix is assumed to be a piezoelectric material whose piezoelectric coefficients and dielectric constants equal zero (or a very small value). In our analysis, the debonding region is taken to be a crack (see figure 2).

Based on the principle of energy balance, the variation of energy in the piezoelectric system for crack growth $d A$ along the friction surface under electromechanical loading is:

$$
d \Pi=-G_{c} d A-d W_{f}
$$

$G_{c}$ is fracture energy, $W_{f}$ is the work done by friction stress during crack growth,

$$
W_{f}=\int_{S_{T}}\left(t+t_{0}\right) v d S
$$

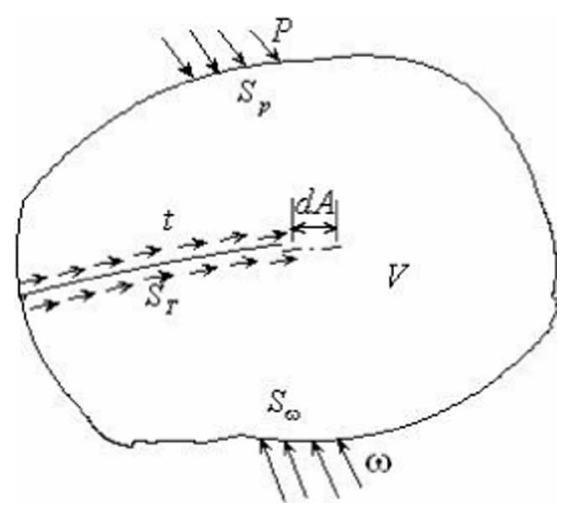

Figure 2. Piezoelectric elastic body with a frictional crack under electromechanical loading. 
$\Pi$ is the generalized mechanical and electrical energy stored inside the piezoelectric body:

$$
\Pi=\frac{1}{2} \int_{\Omega}\left(e+e_{0}\right):\left(s+s_{0}\right) d \Omega-\frac{1}{2} \int_{\Omega}\left(D+D_{0}\right):\left(E+E_{0}\right) d \Omega-\int_{S_{P}} P u d S+\int_{S_{\omega}} \omega \Phi d S
$$

in which $v$ is the relative slip of crack surfaces and $t_{0}$ is the tangential component of pre-stress (or initial stress) on the crack surfaces. $s_{0}, D_{0}, e_{0}$, and $E_{0}$ are selfequilibrium initial stress, electrical displacement, strain and electrical field, respectively, and $s+s_{0}$ and $D+D_{0}$ balance the applied loads.

Using the basic theory of piezoelectricity (1)-(4), one can easily prove the corresponding reciprocal principle of work and the principle of virtual work for piezoelectric material:

$$
\begin{gathered}
\int_{\Gamma} t_{i}^{1} u_{i}^{2} d \Gamma-\int_{\Gamma} \omega^{1} \Phi^{2} d \Gamma+\int_{\Omega} f_{i}^{1} u_{i}^{2} d \Omega=\int_{\Gamma} t_{i}^{2} u_{i}^{1} d \Gamma-\int_{\Gamma} \omega^{2} \Phi^{1} d \Gamma+\int_{\Omega} f_{i}^{2} u_{i}^{1} d \Omega \\
\int_{\Gamma_{t}} \overline{t_{i} \delta} u_{i} d \Gamma+\int_{\Omega} t_{i} \delta u_{i} d \Omega-\int_{\Gamma_{\omega}} \bar{\omega} \delta \Phi d \Gamma-\int_{\Omega} q \delta \Phi d \Omega=\int_{\Omega}\left(\sigma_{i j} \delta \varepsilon_{i j}-D_{i} \delta E_{i}\right) d \Omega
\end{gathered}
$$

Using the two principles, (44) and (45), and following a method similar to that employed for fibre pull-out analysis [14], it can be proved that $U_{t}$ against the incremental debonding length, $l$, is equal to the energy-release rate $G_{i}$ for the debonded crack; that is:

$$
2 \pi a G_{i}=\frac{\partial U_{t}}{\partial l}
$$

in which $U_{t}$ is the total elastic energy and electrical energy stored in the fibre and matrix, which can be expressed in the following form:

$$
\begin{aligned}
U_{t}= & \int_{0}^{l} \int_{0}^{a}\left[\sigma_{f}^{z} \varepsilon_{f}^{z}-D^{z} E^{z}\right] \pi r d r d z+\int_{l}^{L} \int_{0}^{a}\left[\sigma_{f}^{z} \varepsilon_{f}^{z}-D^{z} E^{z}\right] \pi r d r d z \\
& +\int_{0}^{l} \int_{a}^{b}\left[\frac{\left(\sigma_{m}^{z}\right)^{2}}{E_{m}}+\frac{2\left(1+v_{m}\right)}{E_{m}}\left(\tau_{m}^{r z}\right)^{2}\right] \pi r d r d z+\int_{l}^{L} \int_{a}^{b}\left[\frac{\left(\sigma_{m}^{z}\right)^{2}}{E_{m}}+\frac{2\left(1+v_{m}\right)}{E_{m}}\left(\tau_{m}^{r z}\right)^{2}\right] \pi r d r d z
\end{aligned}
$$

Then the following energy criterion is introduced:

$$
G_{i} \geq G_{i c}
$$

in which $G_{i c}$ is a critical interface debonding energy release rate.

In equation (47), $U_{t}$ is a complex function of the material properties of the constituents and geometric factors. Performing the mathematical operation over the debonded and bonded regions for the piezoelectric-epoxy composite system by utilizing a numerical quadrature approach, $G_{i}$ derived in this study can be obtained as a second-order function of the applied stress $\sigma_{a}$ for a fibre/matrix system with given debonding length $l$. 


\section{Numerical calculations and discussion}

To illustrate the effect of electromechanical coupling on the debonding behavior of PFCs and to verify the proposed debonding criterion for the piezoelectric fibre push-out problem, a numerical example is considered for a piezoelectric fibre/epoxy composite system. The parameters of piezoelectric fibre and matrix are given as [4]:

$$
\begin{gathered}
s_{11}=0.019(\mathrm{GPa})^{-1}, s_{33}=0.015(\mathrm{GPa})^{-1}, s_{12}=-0.0057(\mathrm{GPa})^{-1}, \\
s_{13}=-0.0045(\mathrm{GPa})^{-1}, s_{55}=0.039(\mathrm{GPa})^{-1}, d_{33}=390 \times 10^{-12} \mathrm{mV}^{-1}, \\
d_{31}=-d_{15}=-190 \times 10^{-12} \mathrm{mV}^{-1}, g_{33}=24 \times 10^{-3} \mathrm{~V} \mathrm{~m} \mathrm{~N}^{-1}, \\
g_{31}=-11.6 \times 10^{-3} \mathrm{~V} \mathrm{~m} \mathrm{~N}^{-1}, e_{33}=16.25 \times 10^{-9} \mathrm{~N} \mathrm{~V}^{-2}, E_{m}=3 \mathrm{GPa}, v_{m}=0.4
\end{gathered}
$$

The radii of fibre and matrix are given by: $a=0.065 \mathrm{~mm}, b=3 \mathrm{~mm}$ and $l=0.6 \mathrm{~mm}, L=2 \mathrm{~mm}$. The residual fibre clamping stress in the radial direction $q_{0}$ is $-0.1 \mathrm{MPa}$ and $\mu=0.8$ [4]. It should be pointed out that real piezoelectric fibres have a wide variety of shapes and sizes. In general, the radius $a$ of a piezoelectric fibre is about 5-400 $\mu \mathrm{m}$, and the fibre length $L<200 \mathrm{~mm}$ (More detail as to the shape and size of a piezoelectric fibre can be found elsewhere [5, 15-17]). In a real single fibre push-out test, in general, the ideal single fibre composite is one with the value of the matrix radius $b$ being variable, $b \gg a$, and $L$ being between 1 and $2 \mathrm{~mm}$ (see [18] for details).

To illustrate the effect of electrical loading on stress transfer behavior, figure 3 presents distributions of interfacial shear stress as functions of the dimensionless axial distance $z / L$ for partially debonded PFCs in the fibre push-out test. In the calculation, the debonding length is set to be $l=0.6 \mathrm{~mm}$. It is found that the distribution of interfacial shear stress in PFC is similar to that in NPFC nonpiezoelectric fibre composite, in that both show a jump at the point $l / L$ (i.e. the interface between the debonded and bonded regions). It is evident from figure 3 that there is a larger interface shear stress in the debonded region under an applied negative electrical potential. This is because, for a piezoelectric fibre, expansion occurs in the same direction and shrinkage occurs in the transverse direction when the fibre is subjected to an electrical field applied parallel to the polarization direction. For an applied positive electrical potential, the hoop stress induced is in compression, while for a negative applied electrical potential, the hoop stress developed is in tension [19]. Therefore, an applied negative electrical loading leads to a larger shear stress in the debonded region than that induced by a positive electrical loading, according to the Coulomb friction law (11).

The effect of interfacial properties on PFC performance is shown in figure 4, showing the variation of electrical field as a function of axial distance $z / L$ for several values of the parameters of interfacial properties. It can be seen that the electrical field decreases along with either an increase in the residual compressive stress $q_{0}$ or an increase in the Coulomb frictional coefficient $\mu$. These results indicate that, for piezoelectric composites, the interface properties not only control the stress transfer between fibre and matrix but also have an important influence 


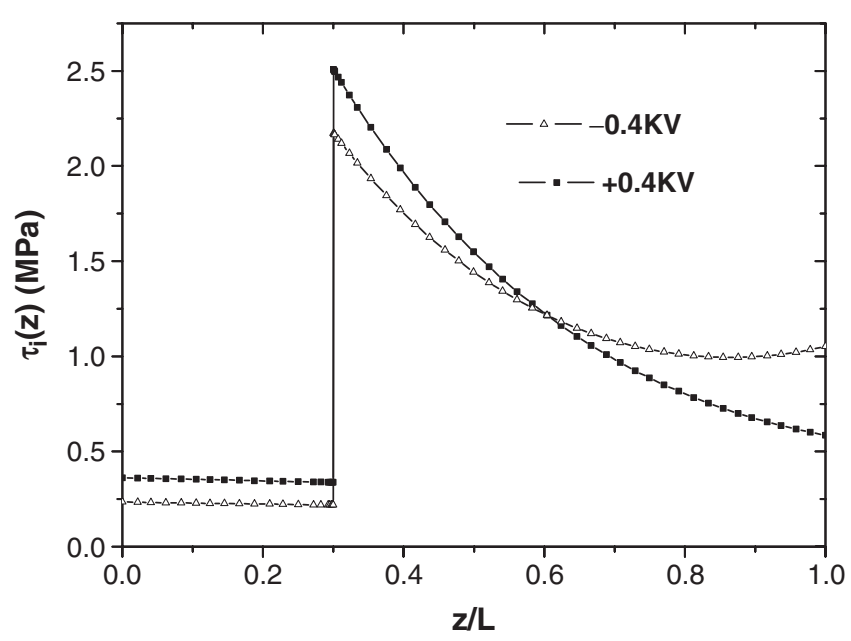

Figure 3. Distribution of interfacial shear stress $\tau_{i}(z)$ under different electrical loading for a constant mechanical loading $\sigma_{a}=0.06 \mathrm{GPa}$.

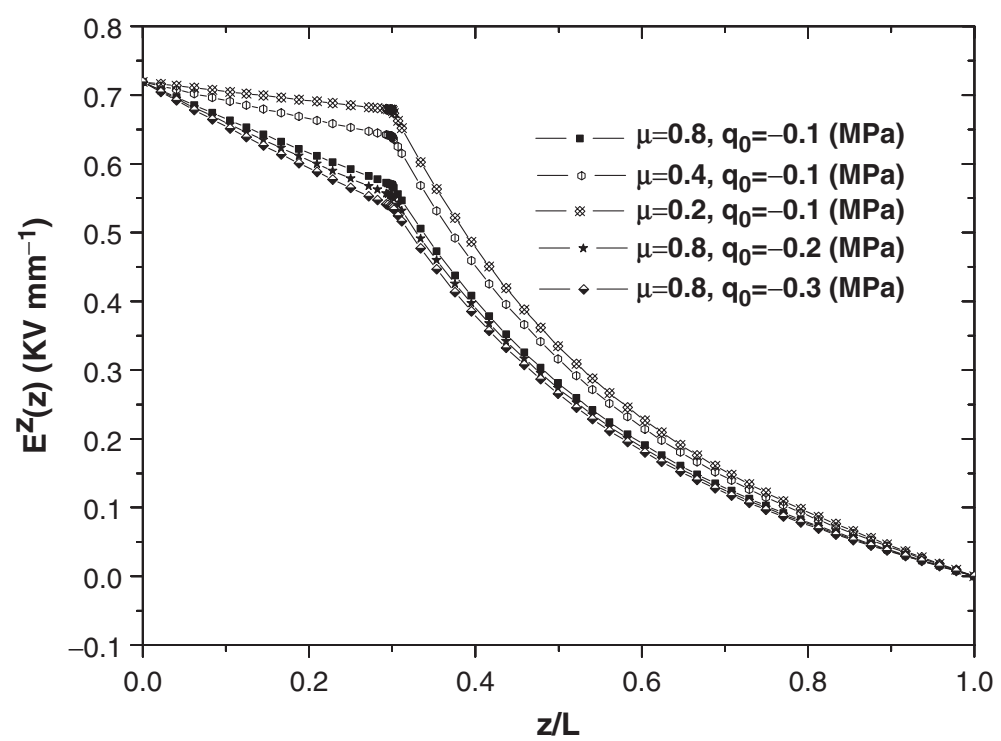

Figure 4. Distribution of electric field in piezoelectric fibre under mechanical loading, $\sigma_{a}=0.06 \mathrm{GPa}$, for different interface properties parameters.

on the distribution of the electrical field. It should be mentioned that at the transition point from the debonded to bonded region there is no jump in electrical field, unlike the case of interfacial shear stress. This phenomenon is very different from the result obtained in [4] and this difference is mainly due to the fact that in our model the expressions for $\sigma_{m}^{r}$ and $\sigma_{m}^{\theta}$ do not include the term, $d \tau_{i} / d z$ owing to the use of Lamè's solution [11]. 


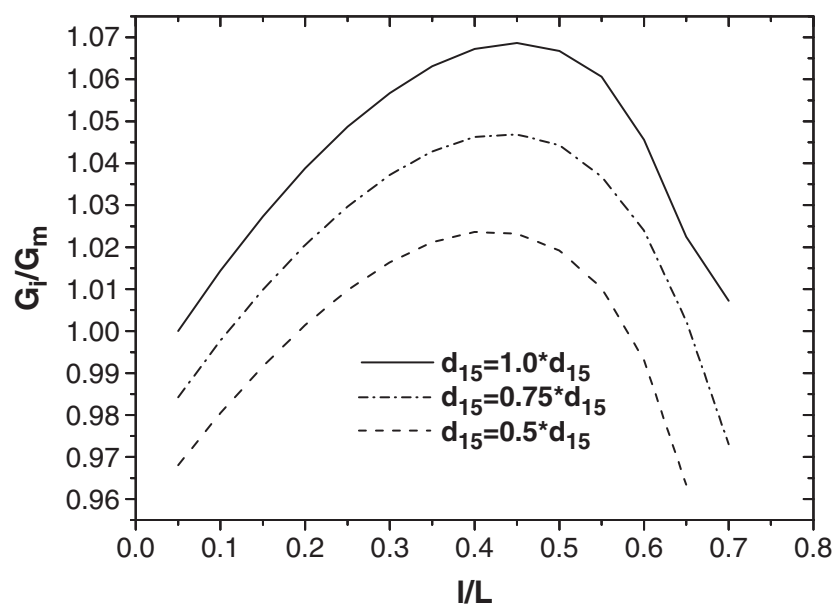

Figure 5. Energy-release rate $G_{i} / G_{m}$ versus $l / L$ under different $d_{15}$ for a constant mechanical loading $\sigma_{a}=0.06 \mathrm{GPa} ; G_{m}$ represents the energy-release rate for $d_{15}=1.0^{*} d_{15}$ and $l / L=0.05$.

To study the effect of the piezoelectric coefficient on the debonding process, the results for the energy release rate are plotted in figure 5 as a function of debonding length $l$ for different piezoelectric coefficients $d_{15}$. From this figure, we can see that the normalized energy-release rate $G_{i} / G_{m}$ increases with an increase in the value of $d_{15}$. In addition, the energy-release rate clearly increases with an increase in the value of $l / L$ until it reaches a maximum. This finding implies that the piezoelectric effect has an important influence on the fibre-debonding process during fibre push-out.

In figure 6, the energy-release rate versus electric potential loading is plotted, from which can be seen that the energy-release rate is linearly dependent on the electrical loading when the PFCs subjected to a constant mechanical loading. The observation above indicates that the total energy-release rate can be used as a debonding criterion for the piezoelectric fibre push-out test.

It should be mentioned that the energy-release rate derived in this paper is the total energy-release rate. Park and Sun [20] obtained the total energy-release rate and the mechanical strain rate for a crack with the electric impermeable condition in an infinite piezoelectric medium for the three fracture modes, theoretically and experimentally. They concluded that the total energy-release rate cannot be used as a fracture criterion because the electrical loading always reduces the total release rate. However, it should be remembered that those results arose from the electrical impermeable condition on the crack surfaces, a condition that is not involved in our problem.

\section{Conclusions}

A theoretical model has been developed for analysing partially debonded PFCs in the push-out test under coupled electrical and mechanical loading. Earlier 


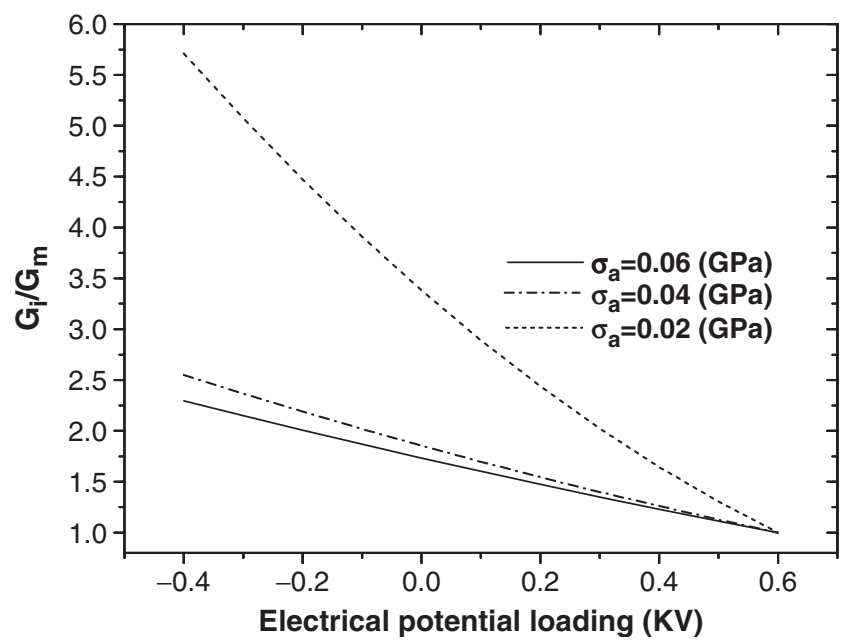

Figure 6. Energy-release rate versus electrical loading under various mechanical loadings, $l / L=0.2 ; G_{m}$ represents the energy release rate for electrical loading at $0.6 \mathrm{kV}$.

work described in [5] applied only to the case of PFCs with a fully bonded or a fully debonded interface under mechanical loading. On the basis of the proposed theoretical model, a debonding criterion including piezoelectric effect has been presented to characterize the stress transfer and debonding processes. Using the proposed theoretical model and energy-release rate, numerical analyses of the effect of interfacial properties, piezoelectric coefficients and electrical loading on stress and electrical field transfer and debond behavior were carried out. The results indicate that both interfacial properties and piezoelectric coefficients have a significant influence on the fibre debonding process. The study also shows that an applied negative electrical potential will cause a larger interface frictional shear stress in debonded regions (see figure 3). Most importantly, the study shows that the energy-release rate can be used as a debonding criterion for piezoelectric fibre push-out.

\section{References}

[1] L.H. He and C.W. Lim, Composites B 34373 (2003).

[2] W. Beckert, W. Kreher, W. Braue, et al., J. Eur. Ceram. Soc. 211455 (2001).

[3] L.J. Nelson, Mater. Sci. Technol. 181245 (2001).

[4] H.Y. Liu, Q.H. Qin and Y.W. Mai, Int. J. Solids Struct. 403511 (2003).

[5] Q.H. Qin, J.S. Wang and Y.L. Kang, Arch. Appl. Mech. In press (2006).

[6] C.H. Hsueh, Mater. Sci. Eng. A 1231 (1990).

[7] C.H. Hsueh, Mater. Sci. Eng. A 12567 (1990).

[8] Y.C. Gao, Y.W. Mai and B. Cotterell, J. Appl. Math. Phys. (ZAMP) 39550 (1988).

[9] L.M. Zhou, Y.W. Mai and Y. Lin, Compos. Eng. 51199 (1995).

[10] M.Y. Quek, Int. J. Adhes. Adhes. 22303 (2002).

[11] X. Zhang, H.Y. Liu, Y.W. Mai, et al., Compos. Sci. Technol. 592179 (1999). 
[12] Q.H. Qin, Fracture Mechanics of Piezoelectric Materials (WIT Press, Southampton, 2001).

[13] Q.H. Qin and S.W. Yu, Int. J. Solids Struct. 34581 (1997).

[14] L.M. Zhou, PhD thesis, University of Sydney (1994), pp. 56-59.

[15] R. Steinhausen, T. Hauke, W. Seifert, et al., J. Eur. Ceram. Soc. 191289 (1999).

[16] L.W. Helen, K.L. Chan and C.L. Choy, Mater. Sci. Eng. B 9929 (2003).

[17] L.J. Nelson, Mater. Sci. Technol. 181245 (2002).

[18] K. Honda and Y. Kagawa, Acta Mater. 443267 (1996).

[19] H.F. Tiersten, Linear Piezoelectric Plate Vibrations (Plenum Press, New York, 1969).

[20] S.B. Park and C.T. Sun, Int. J. Fract. 70203 (1995). 